\title{
乙酰乙酸乙酯在重水和环己烷中的 $\mathrm{C}=\mathrm{O}$ 和 $\mathrm{C}=\mathrm{C}$ 伸缩振动模式的振动 衰减动力学
}

\author{
刘英亮杨帆 王建平* \\ (北京分子科学国家实验室 分子反应动力学实验室 中国科学院化学研究所 北京 100190)
}

\begin{abstract}
摘要 作为典型的 $\beta$-二羰基化合物和 $\alpha, \beta$-烯酮类化合物，乙酰乙酸乙酯在溶液中以多结构存在. 在本文中, 我们利用飞 秒中红外泵浦探测光谱技术, 研究了该化合物在重水和环已烷溶液中不同互变异构体的出现在波长 $6 \mu \mathrm{m}$ (频率范围 $1600 \sim 1800 \mathrm{~cm}^{-1}$ ) 区域的 $\mathrm{C}=\mathrm{O}$ 和 $\mathrm{C}=\mathrm{C}$ 伸缩振动的振动动力学; 并结合稳态红外实验和量子化学计算, 分析了这些吸 收峰的线型特征. 结果表明: 在重水中, 乙酰乙酸乙酯以酮式存在; 而在环已烷中以烯醇式和两种酮式共存. 分析稳态 红外光谱可以看到, 烯醇式结构刚性, 其红外光谱线型主要表现为均匀增宽; 而酮式的线型则兼具均匀增宽和非均匀 增宽. 而且, 即使在同一种溶剂中, 酮式和烯醇式的表观红外光谱线型也有不同. 通过泉浦探测实验获得了 $\mathrm{C}=\mathrm{O}$ 和 $\mathrm{C}=\mathrm{C}$ 伸缩振动衰减动力学和各向异性动力学. 重水中酮式的 $\mathrm{C}=\mathrm{O}$ 的振动能量驰豫过程比其在环已烷中要快, 这可归 因于乙酰乙酸乙酯与溶剂发生的分子间氢键作用. 此外, 烯醇式的 $\mathrm{C}=\mathrm{O}$ 伸缩振动和一些振动模式(如 $\mathrm{COH}$ 面内弯曲) 之间存在费米共振, 直接影响其快速衰减过程, 这也是造成其振动衰减动力学不同于酮式的原因. 各向异性动力学过 程表明, 乙酰乙酸乙酯在重水中的转动速度比其在环已烷中要慢一些, 与它们不同的溶剂化情况有关. 此外, 各向异 性动力学过程表现出量子拍频现象, 在酮式中尤为明显, 意味着相关振动模式存在相干激发.
\end{abstract}

关键词 红外原浦探测; 振动能量驰豫动力学; 各向异性动力学; 乙酰乙酸乙酯

\section{Ultrafast Vibrational Relaxation Dynamics of $\mathrm{C}=\mathrm{O}$ and $\mathrm{C}=\mathrm{C}$ Stretching Modes of Ethyl Acetoacetate in Deuterated Water and Cyclohexane}

\author{
Liu, Yingliang Yang, Fan* Wang, Jianping* \\ (Beijing National Laboratory for Molecular Sciences, Molecular Reaction Dynamics Laboratory, Institute of Chemis- \\ try, Chinese Academy of Sciences, Beijing 100190)
}

\begin{abstract}
As a prototype of both $\beta$-diketone and $\alpha, \beta$-enone compounds, ethyl acetoacetate (EAC) has been known to exist in multiple structures in solutions. In this paper, femtosecond infrared pump-probe spectroscopy was used to examine the vibrational relaxation processes of the $\mathrm{C}=\mathrm{O}$ and $\mathrm{C}=\mathrm{C}$ stretching modes, appeared in the region of $6 \mu \mathrm{m}$ wavelength $(1600 \sim$ $1800 \mathrm{~cm}^{-1}$ in frequency), of the EAC tautomers in deuterated water $\left(\mathrm{D}_{2} \mathrm{O}\right)$ and cyclohexane. Steady-state infrared spectroscopy and quantum chemistry computations were used to understand the characteristics of these vibrational bands and their line shapes. The results showed that in water EAC exists in the keto form, while in cyclohexane it exists in both keto and enol forms, and perhaps in two possible keto forms. As a result of structural rigidity, the steady-state infrared absorption peak is narrow for the enol form, suggesting mainly homogenous broadening; while those of the keto form exhibit the feature of both homogenous and heterogeneous broadenings. The apparent infrared line shapes of the keto and enol forms were somewhat different even in the same solvent. Population dynamics and anisotropy dynamics of the $\mathrm{C}=\mathrm{O}$ and $\mathrm{C}=\mathrm{C}$ stretching mode were obtained and examined by transient infrared pump-probe measurement. In $\mathrm{D}_{2} \mathrm{O}$, the vibrational energy relaxation rate of the $\mathrm{C}=\mathrm{O}$ modes was found to be faster than that in cyclohexane, which can be attributed to the formation hydrogen bond between EAC and solvent molecules. The vibrational relaxation process of the enol form was found to be different from that of the keto form, which can be contributed to Fermi resonance interaction between the $\mathrm{C}=\mathrm{O}$ stretching mode and the $\mathrm{COH}$ bending mode. With the aid of the quantum chemistry calculations, it is concluded that there exists a fast vibrational population equilibrium process between the $\mathrm{C}=\mathrm{O}$ and $\mathrm{C}=\mathrm{C}$ stretching modes and the $\mathrm{COH}$ in-plane bending mode via Fermi resonance. Anisotropy experiments showed a fast orientation dynamics for EAC in cyclohexane than in water, reflecting different solvations. Further, quantum beating was observed in the anisotropy dynamics due to the coherence between the $s s-$ and as-modes, especially for the keto form, suggesting a coherent excitation between the related vibrational modes.
\end{abstract}

Keywords IR pump-probe; vibrational energy relaxation dynamics; anisotropy dynamics; ethyl acetoacetate

*E-mail: jwang@iccas.ac.cn, fanyang@iccas.ac.cn; Tel: 010-62656806, Fax: 010-62563167

Received February 1, 2013; published March 21, 2013.

Project supported by the Knowledge Innovation Program (No. KJCX2-EW-H01), the Hundred Talent Fund from the Chinese Academy of Sciences and the National Natural Science Foundation of China (Nos. 20727001, 91121020 and 21103200).

项目受中国科学院知识创新工程(No. KJCX2-EW-H01)、“百人计划” 和国家自然科学基金(Nos. 20727001,91121020 和 21103200)资助. 


\section{1 引言}

乙酰乙酸乙酯是一种典型的 $\beta$-二羰基化合物. 在溶 液中, 它存在酮式与烯醇式之间的互变异构平衡(图式 $1)$, 该过程受到温度、溶剂和浓度的影响 ${ }^{[1]}$. 在极性溶剂 尤其是氢键溶剂中, 酩式占主导. 而在非极性溶剂中, 它倾向于形成具有分子内氢键的烯醇式, 以降低其自由 能. 在某些溶剂如环己烷和正己烷中, 烯醇式和䣶式共 存. 由于烯醇式有多种顺反异构, 而酮式有易变的构象, 乙酰乙酸乙酯在溶液中的 FTIR 光谱(即线性 IR 光谱)通 常会有吸收峰重叠现象. 如何有效地表征这些结构一直 是个难题.

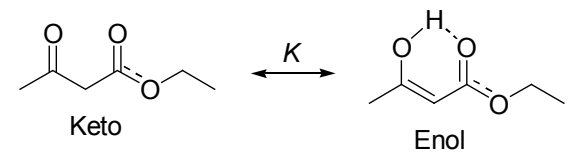

图式 1 乙酰乙酸乙酯的互变异构平衡

Scheme 1 Tautomeric equilibrium of ethyl acetoacetate

分子体系振动态的振动衰减动力学对结构和化学 环境非常敏感, 因此, 可以用来研究该分子体系的超快 结构动力学. 然而, 通过振动衰减动力学来获得结构信 息, 对于多结构共存体系而言, 这显然是个挑战. 乙酰 乙酸乙酯为我们面对这个挑战提供了一个极好的研究 对象.

很多非线性光谱方法都可以用来研究分子体系的 振动衰减动力学, 如红外-泵浦-红外-探测 (IR-pumpIR-probe) 技术 ${ }^{[2]}$, 和红外-泵浦-拉曼-探测 (IR-pumpRaman-probe)技术 ${ }^{[3]}$. 红外-泵浦-拉曼-探测技术的优 点在于可以直接追踪振动态布居数的变化. 但是这种技 术的时间分辨率有限 $(\approx 1 \mathrm{ps})^{[3 \mathrm{~d}]}$, 很难有效追踪更快 $(<$ $1 \mathrm{ps}$ )的布居数变化过程. 此外, 该技术需要较高的红外 洜浦能量以增加瞬态反斯托克斯拉曼信号的强度 ${ }^{[3 \mathrm{~d}, 3 \mathrm{~g}]}$. 而红外-泵浦-红外-探测技术的时间分辨率高(数十 $\mathrm{fs}$ ), 因此可以用于追踪溶液中较快的振动态能量弛豫过程. 而且, 瞬态光谱中的量子拍频信息可以给出振动模式之 间的耦合信息 ${ }^{[4]}$, 有助于指认分子体系的 FTIR 重叠谱 峰. 一个类似化合物 2-乙酰环成酮在四氯化碳溶液中的 二维红外光谱研究的最近报道表明 ${ }^{[5]}$, 酮式结构中对称 和反对称 $\mathrm{C}=\mathrm{O}$ 伸缩振动模式之间存在分子内能量转 移.

由于乙酰乙酸乙酯的烯醇式和酮式的结构差异, 其 振动衰减动力学应存在较大的不同. 但尚未见到关于此 类化合物烯醇式振动衰减动力学的报道. 本工作利用中 红外泉浦探测技术研究了乙酰乙酸乙酯在重水和环己 烷中不同互变异构体中 $\mathrm{C}=\mathrm{O}$ 和 $\mathrm{C}=\mathrm{C}$ 伸缩振动模式的 振动弛豫动力学, 并结合量子化学计算对实验结果进行 了分析.

\section{2 材料与方法}

\section{1 实验方法}

化学纯的乙酰乙酸乙酯(购自 Sinopharm Chemical Reagent)、重水(Cambridge Isotope)、环已烷(Sinopharm) 直接用于实验, 没有进一步提纯. $0.2 \mathrm{~mol} \cdot \mathrm{L}^{-1}$ 的乙酰乙 酸乙酯重水溶液和环已烷溶液置于光程为 $50 \mu \mathrm{m}$ 的由 $\mathrm{CaF}_{2}$ 窗片组成的红外样品池中. 用 Nicolet 6700 傅立叶 变换红外光谱仪采集 FTIR 光谱.

本工作所用的中红外宗浦实验装置与我们最近报 道的相同 ${ }^{[6]}$, 这里仅作简述. 调整飞秒光参量放大器中 的 $\mathrm{BBO}$ 晶体和差频产生器中的 $\mathrm{AgGaS}_{2}$ 晶体的相位匹配 角, 将激光的中心波长调整到 $1680 \mathrm{~cm}^{-1}$, 其光谱的半 峰宽为 $\approx 220 \mathrm{~cm}^{-1}$, 脉冲半峰宽为 $\approx 70 \mathrm{fs}$. 原浦和探测 光的光程差控制范围如下: 在重水溶液中实验时从 $-0.8 \mathrm{ps}$ 到 $6.5 \mathrm{ps}$; 在环己烷溶液中实验时从 $-1.0 \mathrm{ps}$ 到 $6.0 \mathrm{ps}$. 扫描步长为 $10 \mathrm{fs}$. 所有光谱实验均在室温(22 $\left.{ }^{\circ} \mathrm{C}\right)$ 下进行.

\section{2 量子化学计算方法}

利用 Gaussian 03 程序 ${ }^{[7]}$ 中基于密度泛函理论 (Density Functional Theory, DFT)的 B3LYP 方法在 6-31 $++\mathrm{G}^{* *}$ 基组水平上对乙酰乙酸乙酯两种酮式构象和两 种烯醇式构型进行结构优化、非谐性频率分析和 Fermi 共振分析. 从 Fermi 共振分析的结果中, 获得基频模式 与和频模式之间的耦合常数.

\section{3 结果和讨论}

\section{1 线性 IR 光谱}

图 1 给出了乙酰乙酸乙酯在重水和环己烷中的 FTIR 实验光谱和其Voigt 线型拟合曲线. 在图 $1 \mathrm{~A}$ 中, 在 $\mathrm{C}=\mathrm{O}$ 伸缩振动的区域有对应于酮式结构的两个吸收峰. 在图 1B 中有 5 个吸收峰. 其中低频区域的两个谱峰来 自烯醇式，而高频区域的谱峰来自酮式. 这一指认与文 献中报道的类似化合物的指认是一致的 ${ }^{[8]}$. 下面的量化 计算的结果也支持这一指认. 与环已烷溶液中的吸收峰 相比, 酮式在重水中吸收峰明显红移变宽, 这显然与在 两个溶剂中乙酰乙酸乙酯的溶剂化和溶质-溶剂间的氢 键的作用不同有关.

这些主要的谱峰可很好地用 Voigt 线型拟合, 拟合 参数列在表 1 中. 从表 1 发现，不同物种在不同溶剂中 的高斯展宽和洛仑兹展宽的比重存在明显差异. 两种溶 剂中, 酮式结构的红外吸收峰有高斯展宽和洛仑兹展宽 两部分的贡献, 而环已烷中烯醇式的谱峰仅具有洛仑兹 展宽的特征. 这是两种互变异构体的结构差异和溶剂化 差异的光谱表现. 由于酮式骨架的柔性和两个 $\mathrm{C}=\mathrm{O}$ 基 才之间的偶极-偶极相互作用, 使得正则振动模式的频 率存在一个皮秒量级的慢过程. 而且与重水的氢键作用 

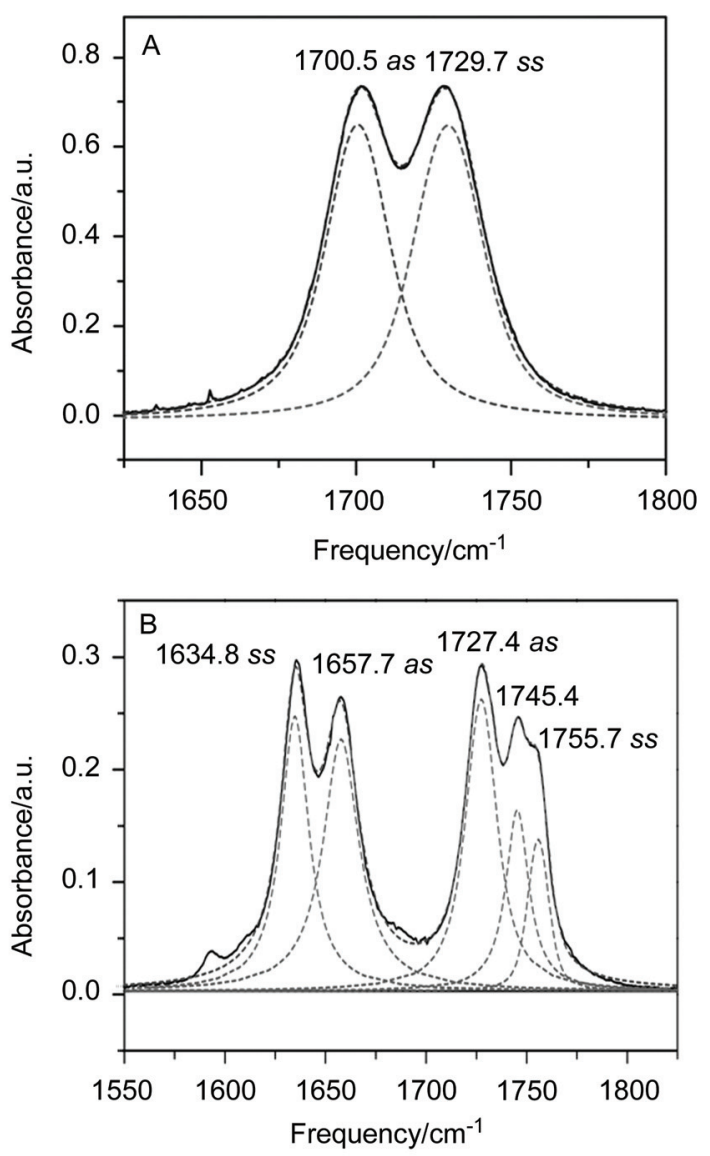

图 1 乙酰乙酸乙酯在重水(A)和环已烷(B)中的 FTIR 光谱(实线)和其 拟合结果(虚线)

Figure 1 FTIR spectra (real line) and their fitting (dashed line) of EAC in water (A) and cyclohexane (B)

的涨落也存在一个皮秒量级上相对较慢的过程 ${ }^{[9]}$, 这也 会对 $\mathrm{C}=\mathrm{O}$ 伸缩振动的非均匀展宽有贡献. 而电子共轭 效应使得烯醇式的结构具有刚性, 这使得非极性溶剂环 己烷的溶剂化结构的涨落对烯醇式振动频率的影响较 小，因此其吸收线型中主要包含均匀展宽组分. 此外, 在环己烷溶液中, 乙酰乙酸乙酯的 IR 光谱(图 1B)出现 了重叠的高频谱峰，表明可能有两种酮式构象.
表 1 乙酰乙酸乙酯在重水和环已烷中 FTIR 光谱的拟合参数 ${ }^{a}$ Table 1 Fitting results of the FTIR experimental data

\begin{tabular}{cccccc}
\hline Solvent & $v_{\mathrm{c}} / \mathrm{cm}^{-1}$ & Area/a.u. & $W_{\mathrm{G}} / \mathrm{cm}^{-1}$ & $W_{\mathrm{L}} / \mathrm{cm}^{-1}$ & $\mathrm{FWHM} / \mathrm{cm}^{-1}$ \\
\hline \multirow{3}{*}{ Water } & 1700.5 & 23.5 & 12.3 & 18.5 & 24.9 \\
& 1729.7 & 24.1 & 16.5 & 16.5 & 27.0 \\
& 1634.8 & 6.5 & 0.0 & 16.9 & 16.9 \\
& 1657.7 & 7.6 & 0.0 & 21.7 & 21.7 \\
Cyclohexane & 1727.4 & 7.2 & 7.4 & 15.7 & 18.8 \\
& 1745.4 & 3.5 & 0.2 & 13.9 & 14.0 \\
& 1755.7 & 2.2 & 9.0 & 6.0 & 12.6 \\
\hline
\end{tabular}

${ }^{a} W_{\mathrm{G}}$ and $W_{\mathrm{L}}$ are line widths of Gaussian and Lorentzian functions respectively; and FWHM is the total full width at half maximum.

为了指认乙酰乙酸乙酯的 FTIR 光谱, 选其在气相 中能量最低的四种结构进行量子化学计算, 优化后的结 构列在图 2 中, 相应的能量、振动模式的特征频率及非 谐性常数列在表 2 中.

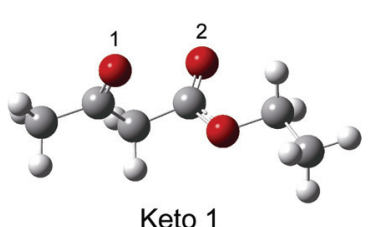

Keto 1

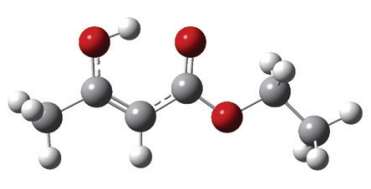

Enol 1
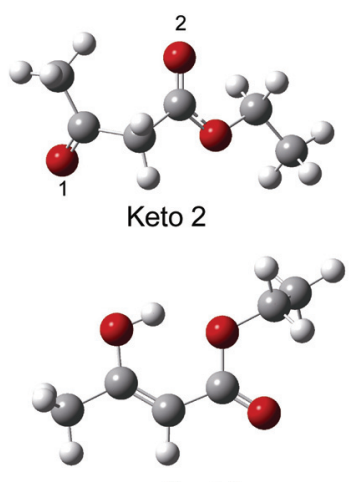

Enol 2
图 2 气相中量子化学优化的乙酰乙酸乙酯的四个低能量的结构

Figure 2 Quantum chemistry optimized four low-energy structures of EAC in gas phase

两个 $\mathrm{C}=\mathrm{O}$ 振动相位相同的模式可以被称为对称伸 缩振动( $s s$ 模式), 而相位相反的模式则可被命名为反对 称伸缩振动模式 ( as 模式), 如图 3 所示. 由于烯醇式中 不存在两个 $\mathrm{C}=\mathrm{O}$, 因此将 $\mathrm{C}=\mathrm{O}$ 和 $\mathrm{C}=\mathrm{C}$ 伸缩振动相

表 2 气相中能量最低的四个结构的相对能量、 $\mathrm{C}=\mathrm{O}$ 和 $\mathrm{C}=\mathrm{C}$ 伸缩振动模式的特征频率、强度和非谐性常数

Table 2 Calculated energies, vibrational frequencies, intensities and anharmonicities of four structures of EAC with lowest energy in gas phase

\begin{tabular}{|c|c|c|c|c|c|}
\hline \multirow{2}{*}{ Species } & \multirow{2}{*}{ Relative energy $/\left(\mathrm{kJ} \bullet \mathrm{mol}^{-1}\right)$} & \multirow{2}{*}{ Frequency $/ \mathrm{cm}^{-1}$} & \multirow{2}{*}{ Intensity $/\left(\mathrm{km} \bullet \mathrm{mol}^{-1}\right)$} & \multicolumn{2}{|c|}{ Anharmonicity $/ \mathrm{cm}^{-1}$} \\
\hline & & & & $\Delta_{\mathrm{ii}}$ & $\Delta_{\mathrm{ij}}$ \\
\hline \multirow{2}{*}{ Enol 1} & \multirow{2}{*}{0.0} & $1626.6 s s^{a}$ & 305.7 & 13.1 & \multirow{2}{*}{19.0} \\
\hline & & 1651.2 as & 393.8 & 10.6 & \\
\hline \multirow{2}{*}{ Enol 2} & \multirow{2}{*}{21.2} & 1637.8 as & 279.9 & 6.5 & \multirow{2}{*}{-0.3} \\
\hline & & 1742.3 ss & 511.3 & 19.5 & \\
\hline \multirow{2}{*}{ Keto 1} & \multirow{2}{*}{11.0} & 1757.3 as & 249.5 & 10.2 & \multirow{2}{*}{19.7} \\
\hline & & $1778.3 s s$ & 203.4 & 9.9 & \\
\hline \multirow{2}{*}{ Keto 2} & \multirow{2}{*}{16.2} & 1743.6 as & 372.6 & 15.5 & \multirow{2}{*}{20.6} \\
\hline & & 1773.6 ss & 105.5 & 32.9 & \\
\hline
\end{tabular}

${ }^{a}$ as is short for the anti-symmetric stretching modes, and $s s$ for the symmetric stretching mode. 
位相同的模式仍然称为 $s s$ 模式, 相位相反的称为 $a s$ 模 式(图 3). 从表 2 给出的数据可以看出在气相中最稳定的 物种为 enol 1 , 它被一个共振辅助分子内氢键稳定 ${ }^{[10]}$. 这与 enol 2 的情形不同, 后者被一个正常氢键所稳定(两 个烯醇式的结构区别在图 2 中给出). 因此 enol 2 的能量 要比 enol 1 高出 $21.2 \mathrm{~kJ} \cdot \mathrm{mol}^{-1}$. 六元环中的氢原子连接 在原乙酰基氧上, 即图 2 中所示的 1 位上, 这与具有对 称结构的 2,4-二酮类化合物明显不同, 后者的氢原子处 在一个对称的双势阱势能面上 ${ }^{[11]}$. 这可能是由于乙氧 基的吸电子效应使得图 2 中所示的 2 位上的氧原子上的 电子密度降低而造成的. 此外, 酮式中反对称伸缩振动 模式出现在对称伸缩振动模式的低频位置, 这是由该结 构中两个局域模的跃迁偶极矩角度和振动耦合常数的 符号共同决定的 ${ }^{[12]}$.

结合量子化学计算结果, 我们可将乙酰乙酸乙酯在 $6 \mu \mathrm{m}$ 区域内的主要 FTIR 吸收峰进行指认. 在重水中位 于 $1700.5 \mathrm{~cm}^{-1}$ 处的峰对应于䣶式的 $a s$ 模式, 1729.7 $\mathrm{cm}^{-1}$ 处的峰对应于 $s s$ 模式. 在环已烷中位于 1634.8 $\mathrm{cm}^{-1}$ 处的峰对应于烯醇式的 $s S$ 模式, $1657.7 \mathrm{~cm}^{-1}$ 处的峰 对应于烯醇式的 $a s$ 模式. 位于 $1727.4 \mathrm{~cm}^{-1}$ 处的峰来自 于酮式的 $a s$ 模式, 而 1745.4 和 $1755.7 \mathrm{~cm}^{-1}$ 处的峰可能 来自两种不同酮式的 ss 模式, 这两种酮式中可能包括能 与烯醇式形成分子间氢键的物种. 与此同时, 只观察到 一个酩式 as 模式的一个可能的原因是偶然简并, 即两 种不同的 as 模式频率非常接近. 此外, 位于 1590,1610 和 $1687 \mathrm{~cm}^{-1}$ 等处的弱谱峰, 其来源目前难以确定.

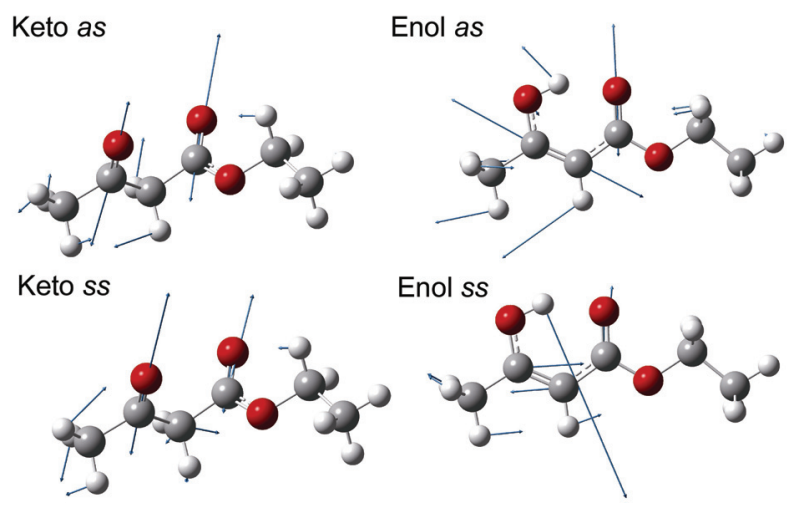

图 3 酮式和烯醇式中 $\mathrm{C}=\mathrm{C}$ 和 $\mathrm{C}=\mathrm{O}$ 的伸缩振动模式

Figure $3 \mathrm{C}=\mathrm{O}$ and $\mathrm{C}=\mathrm{C}$ stretching vibrational modes of the keto form and the enol form

\section{2 泵浦探测瞬态光谱}

乙酰乙酸乙酯在重水和环己烷中若干典型时间延 迟下的魔角偏振瞬态光谱在图 $4 \mathrm{~A}$ 和 $4 \mathrm{~B}$ 中给出. 从图 4A 中可以明显看到, 在重水溶液中乙酰乙酸乙酯的瞬 态光谱表现为两个对应于 $0 \rightarrow 1$ 跃迁的负信号和一个对 应于 $1 \rightarrow 2$ 跃迁的正信号. 而在图 $4 \mathrm{~B}$ 中, 还有烯醇式 $\mathrm{C}=\mathrm{O}$ 和 $\mathrm{C}=\mathrm{C}$ 伸缩振动的瞬态光谱信号. 所有信号的
强度都随延迟增加而衰减. 从图 4B 中可以看出烯醇式 $\mathrm{C}=\mathrm{O}$ 和 $\mathrm{C}=\mathrm{C}$ 伸缩振动的衰减速度要明显慢于酮式, 特别是当时间延迟长于 $4 \mathrm{ps}$ 的时候. 而在 $t<0 \mathrm{ps}$ 时, 检 测到的信号非零，这来自于泵浦光对探测光的自由感应 衰减的调制作用(perturbed free induction decay) ${ }^{[13]}$.
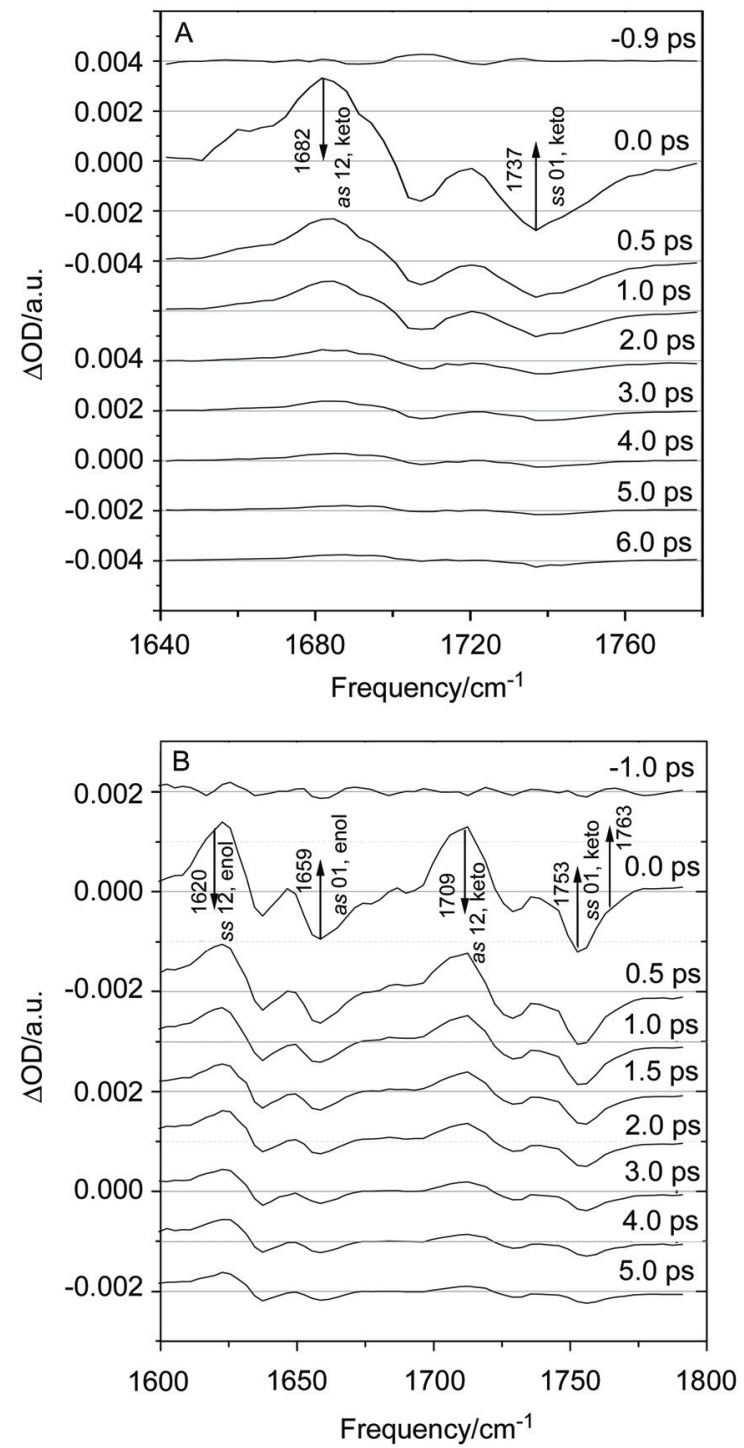

图 4 乙酰乙酸乙酯在重水(A)和环己烷(B)中的几个典型时间延迟下 的百浦探测瞬态光谱

Figure 4 Transient pump-probe spectra of EAC in water (A) and in cyclohexane (B) at selected delay times

\section{3 振动衰减动力学}

为了获得不同物种不同模式的寿命, 追踪了魔角条 件下几个频率位置的运动学轨迹, 并用双指数方程式 (1)对其进行拟合.

$$
\Delta \mathrm{OD}(t)=A_{1} \exp \left(-t / \tau_{1}\right)+A_{2} \exp \left(-t / \tau_{2}\right)+A_{0}
$$

其中, $A_{1}, A_{2}$ 是不同过程的幅度, $A_{0}$ 是终了值, $\tau_{1}, \tau_{2}$ 是相 应过程的时间常数. 原始数据及其拟合曲线如图 5 所示. 拟合所得到的参数在表 3 中给出. 
两种溶剂中酩式 $\mathrm{C}=\mathrm{O}$ 伸缩振动衰减过程的快组分 的时间常数在 $130 \sim 300 \mathrm{fs}$ 范围内. 这一过程由相干效 应导致. 它又分为两种, 当泵浦光和探测光在时间上重 合时的诱导光栅效应 ${ }^{[14]}$ 及被测样品的失相时间大于脉
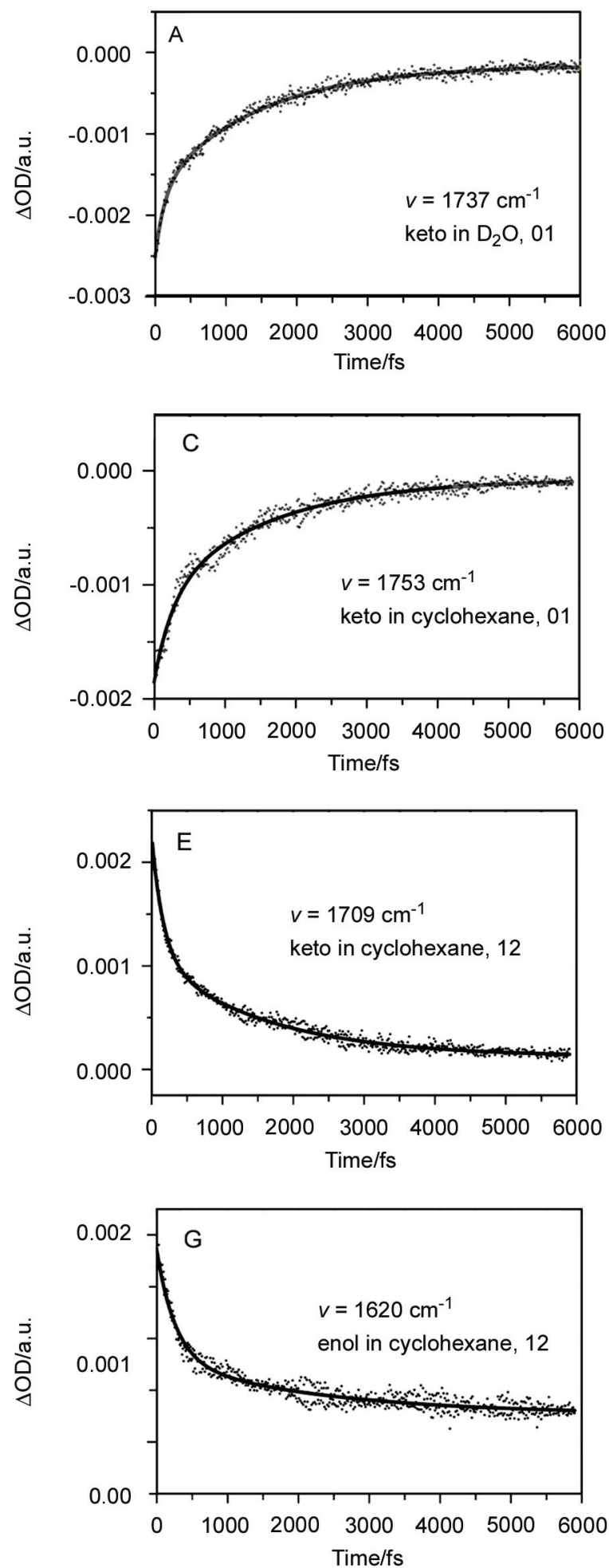

冲持续时间时泵浦光对后来到达样品的探测光的自由 感应衰减的调制作用 ${ }^{[13 a]}$. 而其慢组分在 $1.4 \sim 3.1$ ps 范 围内, 对应于 $v=1$ 态的寿命. 从表 3 中发现, 酮式 $\mathrm{C}=$ $\mathrm{O}$ 伸缩振动的寿命要长于其在环己烷中的寿命, 这意味
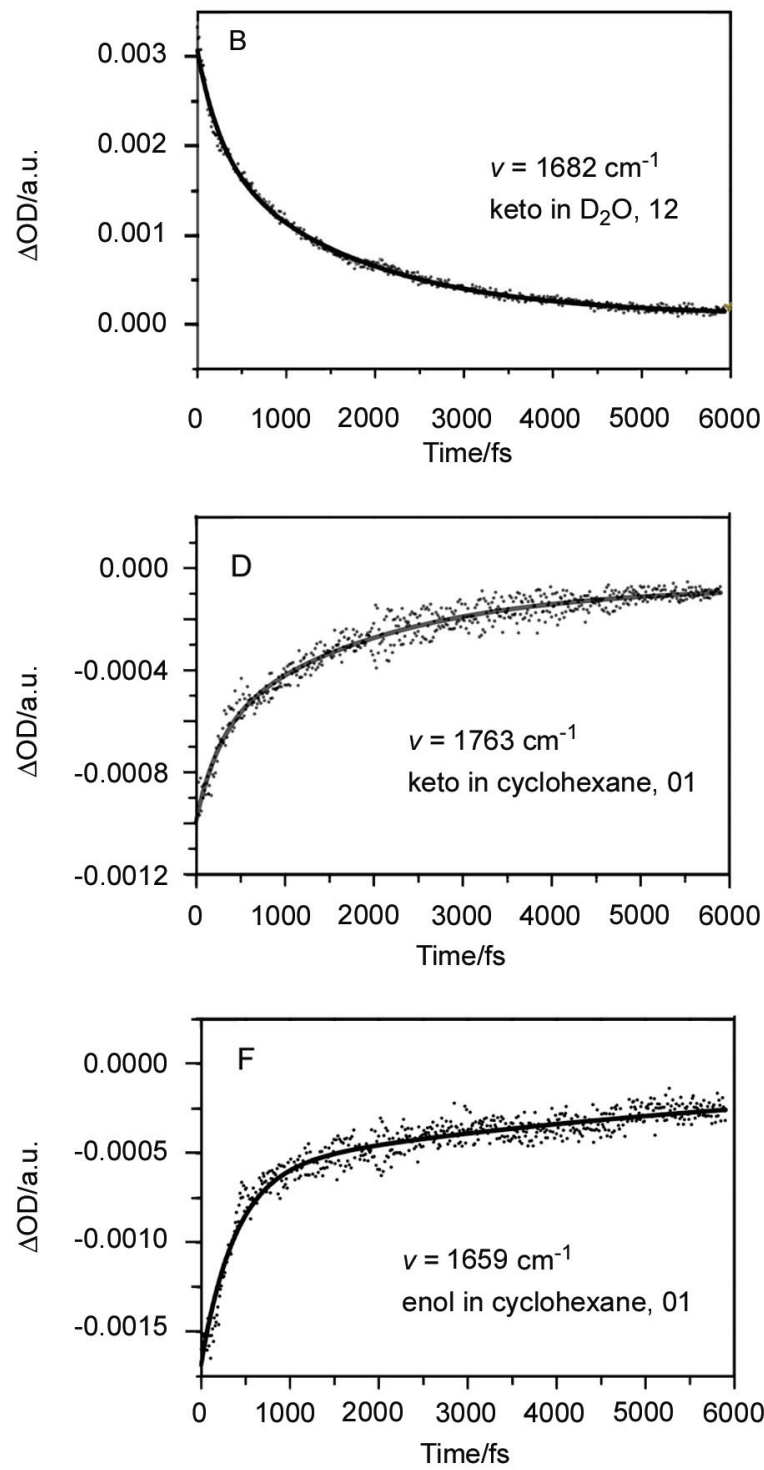

图 5 乙酰乙酸乙酯在重水和环已烷中的 $\mathrm{C}=\mathrm{O}$ 和 $\mathrm{C}=\mathrm{C}$ 振动衰减运动学及其双指数拟合

Figure 5 Population dynamics and their fittings of the $\mathrm{C}=\mathrm{O}$ and $\mathrm{C}=\mathrm{C}$ stretching of $\mathrm{EAC}$ in water and cyclohexane

A, B: keto form in water; C, D and E: keto form in cyclohexane; F, G: enol form in cyclohexane 
表 3 乙酰乙酸乙酯在环已烷和重水中不同探测波长下的振动衰减运动学的双指数拟合参数

Table 3 Double component exponential fitted parameters to the vibration population relaxation process of EAC in water and cyclohexane at specified probing frequencies

\begin{tabular}{|c|c|c|c|c|c|c|c|}
\hline Solvent & Probe frequency $/ \mathrm{cm}^{-1}$ & & $\tau_{1} / \mathrm{fs}$ & $A_{1} / \%$ & $\tau_{2} / \mathrm{fs}$ & $A_{2} / \%$ & $A_{0} / \%$ \\
\hline \multirow[b]{2}{*}{ Water } & 1682 & 12 & $267 \pm 11$ & 32 & $1666 \pm 44$ & 61 & 9 \\
\hline & 1737 & 01 & $137 \pm 9$ & 36 & $1432 \pm 31$ & 60 & 4 \\
\hline \multirow{5}{*}{ Cyclohexane } & 1620 & 12 & $264 \pm 17$ & 57 & $2225 \pm 315$ & 29 & 14 \\
\hline & 1659 & 01 & $192 \pm 14$ & 57 & $3108 \pm 441$ & 32 & 11 \\
\hline & 1709 & 12 & $167 \pm 7$ & 52 & $1598 \pm 53$ & 43 & 5 \\
\hline & 1753 & 01 & $257 \pm 22$ & 38 & $1544 \pm 79$ & 58 & 4 \\
\hline & 1763 & 01 & $265 \pm 32$ & 34 & $1907 \pm 135$ & 59 & 7 \\
\hline
\end{tabular}

着 $\mathrm{C}=\mathrm{O}$ 伸缩振动的能量弛豫过程被其与溶剂氢键作用 所加速, 因为氢键的形成增加了能量受体的态密度 ${ }^{[2,2 k]}$. 在环已烷中酮式的不同频率位置的 $\mathrm{C}=\mathrm{O}$ 伸缩振动寿命 存在明显差异, 这表明 $\mathrm{C}=\mathrm{O}$ 伸缩振动与环境之间的耦 合强度与其溶剂化结构有关. 另外, 烯醇式的 $\mathrm{C}=\mathrm{O}$ 和 $\mathrm{C}=\mathrm{C}$ 伸缩振动衰减过程中的快组分所占的比例 $(\approx$ $57 \%)$ 明显大于酮式中快组分的比例 $(<40 \%)$. 这说明这 个快过程的起源可能不同于酮式的快过程.

根据量化计算的结果, 烯醇式 as 模式与一个和频 模式(1384.5+255.6) $\mathrm{cm}^{-1}$ 存在耦合作用(耦合强度 1.25 $\mathrm{cm}^{-1}$ ), 发生费米共振; 而 ss 模式与两个和频模式存在 耦合, 发生费米共振, 其中一个频率为 $1610.0 \mathrm{~cm}^{-1}$ $\left[(1354.4+255.6) \mathrm{cm}^{-1}\right.$, 耦合强度 $\left.7.90 \mathrm{~cm}^{-1}\right]$, 另一个为 $1618.0 \mathrm{~cm}^{-1}\left[(883.8+737.4) \mathrm{cm}^{-1}\right.$, 耦合强度 -2.70 $\mathrm{cm}^{-1}$ ]. 其中特征频率为 $255.6 \mathrm{~cm}^{-1}$ 的模式是一个骨架 振动模式, 特征频率为 1354.4 和 $1384.5 \mathrm{~cm}^{-1}$ 所对应的 振动模式中都有 $\mathrm{COH}$ 面内弯曲振动的贡献, 883.7 和 $737.4 \mathrm{~cm}^{-1}$ 的振动模式都有六元环中氢原子垂直于环平 面的摆动. 这些直接的机械作用可能使得 $\mathrm{C}=\mathrm{O}$ 和 $\mathrm{C}=$ $\mathrm{C}$ 伸缩振动模式有一个快的振动布居数驰豫过程 ${ }^{[3 \mathrm{a} \sim 3 \mathrm{c}]}$.

\section{4 各向异性动力学}

为了获得跃迁偶极矩的取向信息，我们采集了平行 和垂直偏振下的泵浦探测信号, 并依据 Eq. 2 计算得到 各向异性指数.

$$
S_{\text {aniso }}\left(v_{\text {probe }}, t\right)=\frac{\Delta \mathrm{OD}_{\|}(t)-\Delta \mathrm{OD}_{\perp}(t)}{\Delta \mathrm{OD}_{\|}(t)+2 \Delta \mathrm{OD}_{\perp}(t)}
$$

在乙酰乙酸乙酯中, 由于 $a s$ 和 $s s$ 模式近简并, 在相 干激发的条件下，各向异性指数中包含量子拍 ${ }^{[46,12,15]}$. 因此拟合各向异性动力学时, 利用包含了一个振荡衰减 过程的 Eq. 3:

$$
\begin{aligned}
S_{\text {aniso }}= & A_{1} \cos \left(\frac{2 \pi\left(t-t_{0}\right)}{T}\right) \exp \left(\frac{-\left(t-t_{0}\right)}{t_{1}}\right)+ \\
& A_{2} \exp \left(\frac{-\left(t-t_{0}\right)}{t_{2}}\right)+A_{0}
\end{aligned}
$$

这里 $A_{1}$ 代表振荡衰减过程的初始幅度; $T, t_{1}$ 分别是该振
荡衰减过程的周期和衰减时间常数. $A_{2}, t_{2}$ 代表指数衰减 过程的初始幅度和时间常数, $A_{0}$ 代表实验测到的终了值, 拟合时 $A_{0}$ 均在无穷远处设为零. 拟合曲线和参数分别 在图 6 和表 4 中.

各向异性指数中的慢的指数衰减过程主要对应于 乙酰乙酸乙酯分子在溶液中的跃迁偶极矩的取向扩散 过程. 表 4 中的数据表明环已烷中酮式转动速度 $\left(\mathrm{t}_{2}\right.$ 组 分)明显快于其在重水中的相应速度. 这是由于酮式与 重水之间的氢键作用，使得其转动时不得不带动水合层 的分子. 各向异性指数中快的振荡衰减过程, 由对称伸 缩振动和反对称伸缩振动模式之间的去相干过程导致. 振荡衰减的速度反映了这两个模式去相干过程的速率, 而振荡的周期则反映了这两个模式之间的频率差. 从表 4 中看出, 在探测频率改变时, 振荡周期会跟着改变. 在重水中, 在 $1737 \mathrm{~cm}^{-1}$ 处的振荡周期对应的频率差 $\left(\Delta v=33.3 \mathrm{~cm}^{-1}\right)$ 接近于 FTIR 中两个谱峰的频率差 $\Delta v=$ $29.2 \mathrm{~cm}^{-1}$. 但在 $1682 \mathrm{~cm}^{-1}$ 处对应的 $\Delta v$ 却比两个峰之间 的频率差大. 对于环已烷中的酮式, 发现相应的 $\Delta v$ 分 别与 FTIR 中的两个 $s s$ 模式和 as 模式对应谱峰的频率 差 18.0 和 $28.3 \mathrm{~cm}^{-1}$ 很接近. 这一实验证据定性表明了 这几个谱峰之间的相关性, 亦即确定了这些谱峰是否来 自于同一个结构. 与计算结果比较发现: $18.0 \mathrm{~cm}^{-1}$ 的频 率差与 keto 1 两个模式之间的特征差值 $21.0 \mathrm{~cm}^{-1}$ 相近, 而 $28.3 \mathrm{~cm}^{-1}$ 则与 keto 2 中的两个模式的频率差 29.0 $\mathrm{cm}^{-1}$ 相近. 这表明: 环己烷溶液中酮式两种构象的结构 与图 2 中的 keto 1 和 keto 2 的结构相近. 因此, 它们可 能来源于两种不同的构象的酮式. 然而, 这里并不能够 完全排除与烯醇式的分子间氢键作用导致的峰䢃裂.

\section{4 结论}

在本工作中, 我们利用 6- $\mu \mathrm{m}$ IR 泵浦探测技术并结 合 FTIR 和量子化学计算, 研究了乙酰乙酸乙酯在重水 和环已烷中的结构和振动动力学. 研究表明, 该化合物 的结构和动力学受溶剂影响显著. 在这两种溶剂中乙酰 乙酸乙酯的平衡构型有很大的差异: 在重水中, 只有酮 

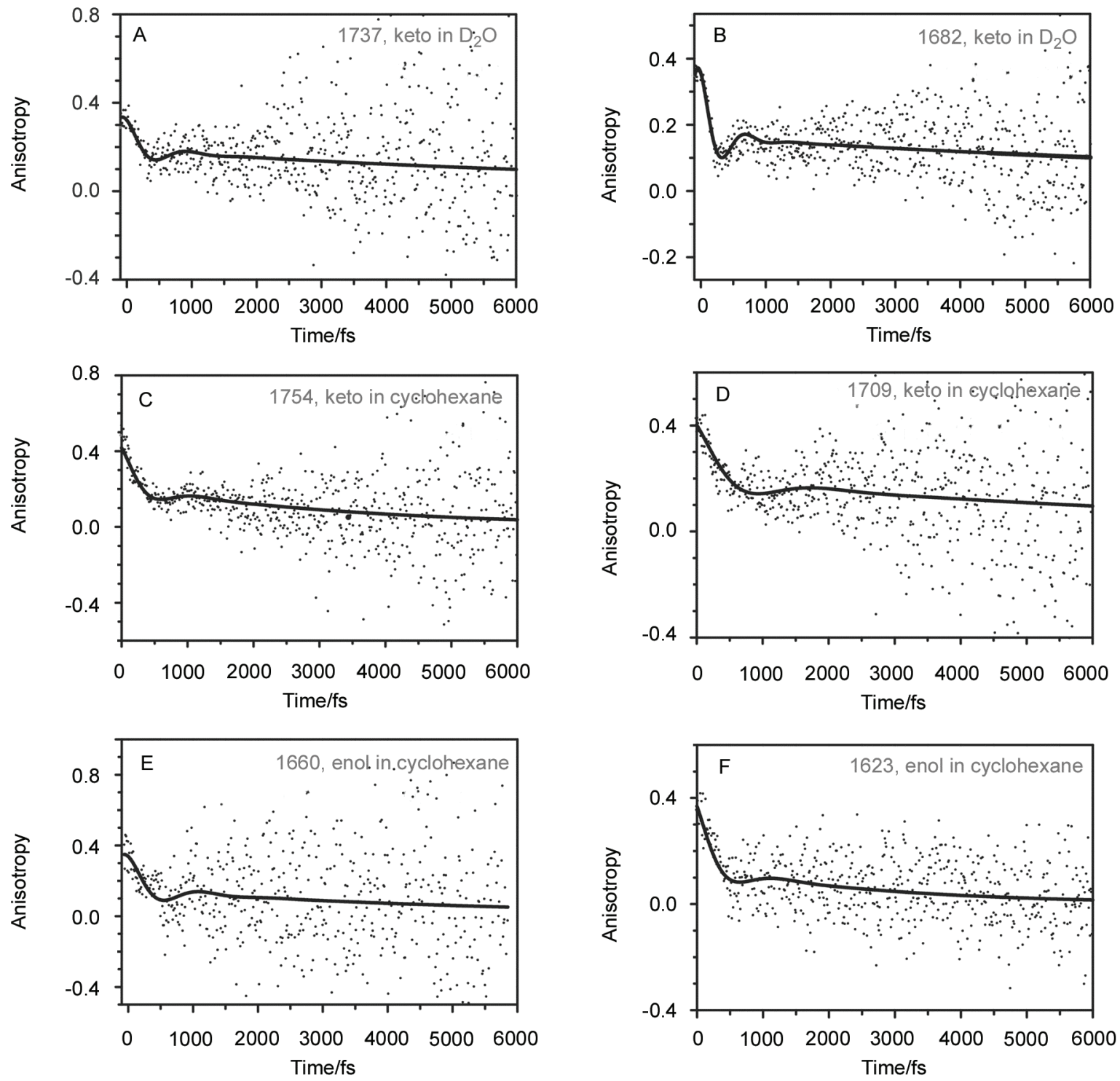

图 6 乙酰乙酸乙酯的各向异性动力学和其拟合曲线

Figure 6 Fitted results of anisotropy dynamics of EAC in water

A, B: keto form in water; C, D: keto form in cyclohexane; E, F: enol form in cyclohexane

表 4 乙酰乙酸乙酯在重水和环已烷中的各向异性运动学的拟合参数

Table 4 Fitted results of anisotropy dynamics of EAC in water and cyclohexane

\begin{tabular}{cccccccc}
\hline Solvent & Probe frequency $/ \mathrm{cm}^{-1}$ & $A_{1}$ & $t / \mathrm{fs}$ & $\Delta v^{a} / \mathrm{cm}^{-1}$ & $t_{1} / \mathrm{fs}$ & $A_{2}$ & $t_{2} / \mathrm{fs}$ \\
\hline \multirow{2}{*}{ Water } & 1682 & 0.19 & $732 \pm 64$ & 45.5 & $289 \pm 66$ & 0.16 & $12626 \pm 2392$ \\
& 1737 & 0.19 & $1001 \pm 305$ & 33.3 & $372 \pm 293$ & 0.19 & $9284 \pm 3246$ \\
& 1623 & 0.22 & $1457 \pm 262$ & 22.9 & $345 \pm 111$ & 0.15 & $2677 \pm 500$ \\
Cyclohexane & 1660 & 0.18 & $1184 \pm 586$ & 28.2 & $438 \pm 396$ & 0.15 & $5327 \pm 2800$ \\
& 1709 & 0.20 & $2126 \pm 418$ & 15.7 & $556 \pm 187$ & 0.20 & $8266 \pm 2352$ \\
& 1754 & 0.20 & $1299 \pm 774$ & 25.7 & $360 \pm 233$ & 0.22 & $3464 \pm 751$ \\
\hline
\end{tabular}

${ }^{a}$ Calculated from the period of the oscillation decay.

式; 而在环己烷中, 烯醇式和酮式共存, 且酮式可能以 两种形式存在; 烯醇式也以有共振辅助分子内氢键的结 构存在.
两种溶剂中乙酰乙酸乙酯的振动衰减动力学通过 瞬态光谱测量中获得. 研究发现, 酮式在重水中较在环 己烷中有一个相对短的寿命, 这一点说明氢键作用的存 
在加速了 $\mathrm{C}=\mathrm{O}$ 伸缩振动的衰减过程; 此外还发现在环 己烷中振动能量衰减的速率与溶质分子的溶剂化密切 相关. 各向异性动力学中快的振荡衰减过程是由反对称 伸缩振动模式和对称伸缩振动模式之间的快速去相干 过程导致, 而其慢组分则主要由分子整体的转动导致. 实验表明, 乙酰乙酸乙酯在重水中的转动速度要明显慢 于在环已烷中的转动速度.

结合量子化学计算, 获得了烯醇式中 $\mathrm{C}=\mathrm{O}$ 和 $\mathrm{C}=$ $\mathrm{C}$ 伸缩振动的衰减路径. 与 $\mathrm{COH}$ 的直接氢键作用, 使得 烯醇式中的 $\mathrm{C}=\mathrm{O}$ 伸缩振动模式与一个和频模式发生费 米共振, 经历一个几百飞秒的快衰减过程, 和一个皮秒 尺度的慢衰减过程.

\section{References}

[1] (a) Powling, J.; Bernstein, H. J. J. Am. Chem. Soc. 1951, 73, 4353; (b) Burdett, J. L.; Rogers, M. T. J. Am. Chem. Soc. 1964, 86, 2105; (c) Rogers, M. T.; Burdett, J. L. Can. J. Chem. 1965, 43, 1516; (d) Drexler, E. J.; Field, K. W. J. Chem. Educ. 1976, 53, 392; (e) Emsley, J.; Ernst, R.; Hathaway, B.; Warren, K.; Emsley, J. Structure \& Bonding, Springer, Berlin/Heidelberg, 1984, Vol. 57, p. 147; (f) Cook, A. G.; Feltman, P. M. J. Chem. Educ. 2007, 84, 1827.

[2] (a) Berkerle, J. D.; Casassa, M. P.; Cavanagh, R. R.; Heilweil, E. J.; Stephenson, J. C. J. Chem. Phys. 1989, 90, 4619; (b) Piatkowski, L.; Bakker, H. J. J. Phys. Chem. A 2010, 114, 11462; (c) Kropman, M. F.; Nienhuys, H.-K.; Woutersen, S.; Bakker, H. J. J. Phys. Chem. A 2001, 105, 4622; (d) Lock, A. J.; Woutersen, S.; Bakker, H. J. J. Phys. Chem. A 2001, 105, 1238; (e) Heilweil, E. J. J. Chem. Phys. 1986, 85, 5004; (f) Grubbs, W. T.; Dougherty, T. P.; Heilweil, E. J. J. Phys. Chem. 1995, 99, 10716; (g) Arrivo, S. M.; Kleiman, V. D.; Grubbs, W. T.; Dougherty, T. P.; Heilweil, E. J. Laser Chem. 1999, 19, 1; (h) Lim, M.; Hochstrasser, R. M. J. Chem. Phys. 2001, 115, 7629; (i) Nibbering, E. T. J.; Elsaesser, T. Chem. Rev. 2004, 104, 1887; (j) Banno, M.; Ohta, K.; Tominaga, K. J. Phys. Chem. A 2008, 112, 4170; (k) Banno, M.; Ohta, K.; Yamaguchi, S.; Hirai, S.; Tominaga, K. Acc. Chem. Res. 2009, 42, 1259; (1) Banno, M.; Ohta, K.; Tominaga, K. Phys. Chem. Chem. Phys. 2012, 14, 6359; (m) Shaw, D. J.; Panman, M. R.; Woutersen, S. Phys. Rev. Lett. 2009, 103, 227401; (n) Yang, M.; Szyc, Ł.; Dreyer, J.; Nibbering, E. T. J.; Elsaesser, T. J. Phys. Chem. A 2010, 114, 12195.

[3] (a) Deàk, J. C.; Iwaki, L. K.; Dlott, D. D. Chem. Phys. Lett. 1998, 293, 405; (b) Pein, B. C.; Seong, N.-H.; Dlott, D. D. J. Phys. Chem. A 2010, 114, 10500; (c) Seong, N.-H.; Fang, Y.; Dlott, D. D. J. Phys. Chem. A 2009, 113, 1445; (d) Dana, D.; John, D.; Stuart, R.; Law- rence, I. In Ultrafast Infrared And Raman Spectroscopy, CRC Press, New York, 2001; (e) Dlott, D. D. Chem. Phys. 2001, 266, 149; (f) Fang, Y.; Shigeto, S.; Seong, N.-H.; Dlott, D. D. J. Phys. Chem. A 2008, 113, 75; (g) Deàk, J. C.; Iwaki, L. K.; Dlott, D. D. Opt. Lett. 1997, 22, 1796 .

[4] (a) Yamaguchi, S.; Banno, M.; Ohta, K.; Tominaga, K.; Hayashi, T. Chem. Phys. Lett. 2008, 462, 238; (b) Collini, E.; Scholes, G. D. J. Phys. Chem. A 2009, 113, 4223.

[5] Park, S.; Ji, M. ChemPhysChem 2011, 12, 799.

[6] Yang, F.; Liu, Y.-L.; Wang, J.-P. Acta Phys. Chim. Sin. 2012, 28, 759. (杨帆, 刘英亮, 王建平, 物理化学学报, 2012, 28, 759).

[7] Frisch, M. J.; Trucks, G. W.; Schlegel, H. B.; Scuseria, G. E.; Robb, M. A.; Cheeseman, J. R.; Vreven, J. T.; Kudin, K. N.; Burant, J. C.; Millam, J. M.; Iyengar, S. S.; Tomasi, J.; Barone, V.; Mennucci, B.; Cossi, M.; Scalmani, G.; Rega, N.; Petersson, G. A.; Nakatsuji, H.; Hada, M.; Ehara, M.; Toyota, K.; Fukuda, R.; Hasegawa, J.; Ishida, M.; Nakajima, T.; Honda, Y.; Kitao, O.; Nakai, H.; Klene, M.; Li, X.; Knox, J. E.; Hratchian, H. P.; Cross, J. B.; Adamo, C.; Jaramillo, J.; Gomperts, R.; Stratmann, R. E.; Yazyev, O.; Austin, A. J.; Cammi, R.; Pomelli, C.; Ochterski, J. W.; Ayala, P. Y.; Morokuma, K.; Voth, G. A.; Salvador, P.; Dannenberg, J. J.; Zakrzewski, V. G.; Dapprich, S.; Daniels, A. D.; Strain, M. C.; Farkas, O.; Malick, D. K.; Rabuck, A. D.; Raghavachari, K.; Foresman, J. B.; Ortiz, J. V.; Cui, Q.; Baboul, A. G.; Clifford, S.; Cioslowski, J.; Stefanov, B. B.; Liu, G.; Liashenko, A.; Piskorz, P.; Komaromi, I.; Martin, R. L.; Fox, D. J.; Keith, T.; Al-Laham, M. A.; Peng, C. Y.; Nanayakkara, A.; Challacombe, M.; Gill, P. M. W.; Johnson, B.; Chen, W.; Wong, M. W.; Gonzalez, C.; Pople, J. A.; Gaussian, Inc., Wallingford CT, 2003.

[8] (a) Gren, E. Y.; Grinvalde, A. K.; Vanag, G. Y. J. Appl. Spectrosc. 1967, 6, 253; (b) Gren, E. Y.; Grinvalde, A. K.; Vanag, G. Y. J. Appl. Spectrosc. 1967, 6, 146.

[9] (a) Asbury, J. B.; Steinel, T.; Kwak, K.; Corcelli, S. A.; Lawrence, C. P.; Skinner, J. L.; Fayer, M. D. J. Chem. Phys. 2004, 121, 12431; (b) Ghosh, A.; Hochstrasser, R. M. Chem. Phys. 2011, 390, 1; (c) Loparo, J. J.; Roberts, S. T.; Tokmakoff, A. J. Chem. Phys. 2006, 125, 194522.

[10] (a) Gilli, G.; Bellucci, F.; Ferretti, V.; Bertolasi, V. J. Am. Chem. Soc. 1989, 111, 1023; (b) Bertolasi, V.; Gilli, P.; Ferretti, V.; Gilli, G. J. Am. Chem. Soc. 1991, 113, 4917; (c) Gilli, P.; Bertolasi, V.; Ferretti, V.; Gilli, G. J. Am. Chem. Soc. 2000, 122, 10405; (d) Dannenberg, J. J.; Rios, R. J. Phys. Chem. 1994, 98, 6714

[11] (a) Hargis, J. C.; Evangelista, F. A.; Ingels, J. B.; Schaefer, H. F. J. Am. Chem. Soc. 2008, 130, 17471; (b) Belot, J. A.; Clark, J.; Cowan, J. A.; Harbison, G. S.; Kolesnikov, A. I.; Kye, Y.-S.; Schultz, A. J.; Silvernail, C.; Zhao, X. J. Phys. Chem. B 2004, 108, 6922.

[12] Hochstrasser, R. M. Chem. Phys. 2001, 266, 273.

[13] (a) Hamm, P. Chem. Phys. 1995, 200, 415; (b) Likforman, J. P.; Joffre, M.; Chériaux, G.; Hulin, D. Opt. Lett. 1995, 20, 2006.

[14] Hegarty, J.; Sturge, M. D. J. Opt. Soc. Am. B 1985, $2,1143$.

[15] Smith, E. R.; Jonas, D. M. J. Phys. Chem. A 2011, 115, 4101. 